Original Article

\title{
Effects of the flexion-distraction technique and drop technique on straight leg raising angle and intervertebral disc height of patients with an intervertebral disc herniation
}

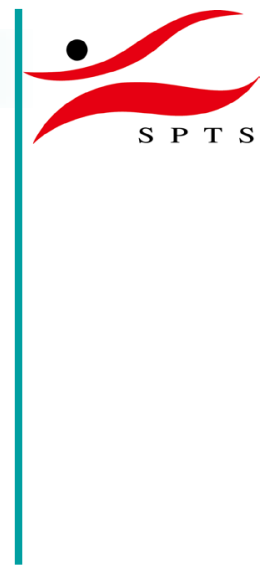

\author{
Hyunju Oh, PhD, PT ${ }^{1)}$, SeokJoo Choi ${ }^{1)}$, Sangyong Lee, PhD, $\mathrm{PT}^{2)}$, Jioun Choi, PhD, PT ${ }^{3)}$, \\ Kwansub LeE, PhD, $\mathrm{PT}^{4)^{*}}$ \\ 1) Department of Physical Therapy, Taegu Science University, Republic of Korea \\ 2) Department of Physical Therapy, U1 University, Republic of Korea \\ 3) Department of Physical Therapy, Shin Orthopedic Hospital, Republic of Korea \\ 4) Department of Physical Therapy, Daegu Health College: 15 Taejeondong, Yeongsongro, Buk-gu, \\ Daegu 41453, Republic of Korea
}

\begin{abstract}
Purpose] The purpose of this study was to determine the effects of the flexion-distraction technique and drop technique on the straight leg raising angle and intervertebral disc height of patients with an intervertebral disc herniation. [Participants and Methods] Thirty patients with herniated intervertebral discs were divided into either an experimental group ( $\mathrm{n}=15)$, who underwent flexion-distraction and drop techniques, or a control group $(\mathrm{n}=15)$, who obtained spinal decompression therapy. Both groups were treated three times per week for eight weeks. [Results] An intragroup comparison showed that the straight leg raising angle and the intervertebral disc height significantly increased in both groups. But it was not significantly difference in the intergroup comparison. [Conclusion] The flexion-distraction technique and the drop technique may serve as effective interventions for the straight leg raising angle and intervertebral disc height in patients with intervertebral disc herniations.

Key words: Flexion-Distraction technique, Drop technique, Disc height
\end{abstract}

(This article was submitted Apr. 5, 2019, and was accepted May 27, 2019)

\section{INTRODUCTION}

Lumbar pain is a common disorder and one of the main concerns in physical therapy, with a $70 \%$ overall prevalence rate in developed countries ${ }^{1}$. Conservative treatments for intervertebral disc herniations include medication, bed rest, physical therapy, massage, kinesitherapy, manual therapy, and traction therapy ${ }^{2}$. One type of manual therapy is chiropractic treatment, which includes the flexion-distraction and drop techniques.

The flexion-distraction technique is used clinically by more than $50 \%$ of chiropractors. It broadens the distance between the spinous processes to widen the space around the disc. This creates a negative pressure within the space, which pulls the bulged disc back into the spine and can place the subluxed vertebra back where it should $\mathrm{be}^{3)}$. This technique can be used for traction of specific areas of the lumbar vertebrae or for intensive movement of apophyseal joints to extend the anterior and posterior longitudinal ligaments to rearrange the intervertebral discs ${ }^{4}$. Gay et al. ${ }^{5}$ reported the technique was safe and physiologically effective for patients with lumbar pain and chronic pelvic pain.

The drop technique - mainly used to realign the pelvis, sacrum, spine, and neck bones - is a system in which solid joints, such as the pelvic joints, can be safely treated using a specially designed table. It is considered the most commonly used

*Corresponding author. Kwansub Lee (E-mail: pt1026@nate.com)

(C2019 The Society of Physical Therapy Science. Published by IPEC Inc.

(c) (i) $\odot$ This is an open-access article distributed under the terms of the Creative Commons Attribution Non-Commercial No Deriva-

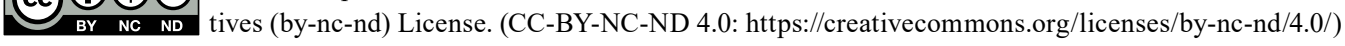


chiropractic technique ${ }^{6}$. Therapists use a unique analysis of the Thompson technique to precisely locate and treat main subluxations. In developed countries, such as the United States and Canada, patients with lumbar pain prefer chiropractic care the most. Patients who have undergone this care because of lumbar pain have been shown to be three times more satisfied than those who have received general medical treatments ${ }^{7}$. Although the flexion-distraction and drop techniques have been widely used for a variety of spinal disorders, their therapeutic effects have not been clearly established.

In this context, we studied, compared, and analyzed the effects of the flexion-distraction and drop techniques-therapies usually applied to patients with herniated intervertebral discs - and spinal decompression therapy to determine their clinical effects and provide support for their scientific basis.

\section{PARTICIPANTS AND METHODS}

This study included 30 female patients from 20 to 60 years of age who were admitted to or visited an orthopedic clinic in Daegu, South Korea, because of lumbar pain lasting at least three months. Their symptoms were consistent with those of herniated intervertebral discs from L5-S1 in terms of clinical findings confirmed by orthopedic specialists and pain complaints, neurological patterns, and radiological findings confirmed by attending physicians with medical devices, including X-rays, computed tomography (CT) scans, and magnetic resonance imaging (MRI) scans. The experimental group underwent both the flexion-distraction and drop techniques $(\mathrm{EG}, \mathrm{n}=15)$ and had an average age of $38.5 \pm 7.8$ years, were $161.4 \pm 5.5 \mathrm{~cm}$ in height, and $56.2 \pm 7.8 \mathrm{~kg}$ in weight. The control group underwent the spinal decompression therapy (CG, $\mathrm{n}=15$ ) and had an average age of $37.4 \pm 10.7$ years, were $160.2 \pm 6.3 \mathrm{~cm}$ in height, and $55.8 \pm 9.4 \mathrm{~kg}$ in weight. There was no significant difference in the homogeneity between the two groups. Ethical approval for this study was granted by the U1 University institutional review board. All patients read and signed consent forms, in accordance with the ethical standards of the Declaration of Helsinki. While those who had symptoms for more than one week or had not undergone a decompression and manual therapy were included, those who had a surgical history for lumbar vertebra, had a spinal tumor, an intervertebral disc infection, or had a contraindication for manual therapy were excluded from this study.

Zenith-Cox flexion tables (Zenith-100, USA) for the flexion-distraction technique, drop tables (Zenith 440, USA) for the drop technique, and devices for spinal decompression therapy (MID 4 M Series, WIZ medical, KOR) for the control group were used by physical therapists with more than 10 years of clinical experience. In addition, both groups were given conservative physical therapies, including hot packs (20 minutes), interferential current therapy (100 bps, 15 minutes), and ultrasound (5 minutes). All treatments were provided three times per week for eight weeks.

For the flexion-distraction technique, a therapist firmly put one of his/her thenars on the spinous process of the lumbar to be treated and held on to a handle at the tail of the Cox table. The therapist then pressed down on the caudal pelvic section of the table, performing a four-to-five-second flexion-distraction movement five times to exert a total of 20 seconds of distraction. The caudal section was pressed down by no more than $5 \mathrm{~cm}$. When one set consisted of five repeated flexion-distractions to reach 20 seconds, three sets were applied to each patient. After finishing the flexion-distraction technique, the therapist performed a foramen magnum pump 10 times by supporting and fixing the back of the patient's head with one hand and pressing down on the table's tail to apply a flexion-distraction procedure.

For the drop technique, the lengths of the patient's pelvis and legs were determined and there was a determination for the presence of cervical syndrome. A patient who had a subluxation of the lumbar and pelvis was asked to lie prone for the technique. For the positive Derifield adjustment, the therapist put the thenar of his/her dominant hand on the posterior superior iliac spine of the patient, who was in a prone position, and the other hand on the ischial tuberosity of the opposite side. When the direction of dropping the pelvis was set up to be anteroinferior, the adjustment was performed three to four times from the rear to the front and from lower to upper. For the negative Derifield adjustment, the therapist put the thenar of his/her dominant hand on the ischial tuberosity of the patient, who was in a prone position, and held the wrist by his/her other hand and performed the adjustment three to four times from lower to upper. The therapist then put his/her rear lower forearm on the front of the patient's thigh and the caput ulnae of his/her upper forearm on the medial portion of the posterior superior iliac spine of the patient, locked his/her fingers together, and raised one leg of the patient to slightly adduct it for adjustment. After adjusting the pelvic subluxation, the sacrum subluxation was adjusted by setting up the tension of the table to be weak. The adjustment range in the spinal column was limited to the lumbar and pelvic areas.

For the spinal decompression therapy, with the patient lying in a supine position on the device, the therapist used the air grip extension to lock air belts around the pelvic and thoracic areas. The therapist then fixed the head with the head strap to avoid a slip on the attachment part and applied the sacrum extension to maintain the lumbar lordosis. The therapy was performed for 20 minutes with the ratio of hold time to rest time set at 2:1.

For an estimation of the straight leg raising angle, straight leg raising was checked with ankle joint dorsiflexion. When the axis was fixed on the greater trochanter of the intertrochanteric crest, the fixed arm was parallel to the lateral centerline of the trunk. When the moving arm was parallel to the lateral centerline of the intertrochanteric crest, the angle that lower extremity radiating pain occurred under the thighs and knee joints was measured by a therapist with a goniometer.

To estimate the intervertebral disc height, the Picture Archiving and Communication System (PACS) was used to measure the height with radiology equipment (DS-20UR, KOR). The radiography was performed only in the morning to reduce measurement errors and without any weight bearing to exclude changes in lordosis and kyphosis angles on the sagittal plane 
and changes in intervertebral disc height due to weight bearing. The height of the intervertebral disc (L5-S1) was measured with the method described by Inoue et al. ${ }^{8}$ ) This method involves adding the heights of the disc measured from the front, middle, and rear, and then dividing the value by three. The heights were measured by one radiographer who did not know the symptoms and data of the patients.

For statistical analyses, an intragroup comparison was performed with a paired t-test, an intergroup comparison was performed with an independent t-test, and the significance level $(\alpha)$ was set at 0.05 .

\section{RESULTS}

The results of the intragroup comparison showed that the straight leg raising angle and the intervertebral disc height significantly increased in both the EG and the CG groups $(\mathrm{p}<0.05)$. But it was not significantly difference in the intergroup comparison $(\mathrm{p}>0.05)$ (Table 1$)$.

\section{DISCUSSION}

Kwon et al. ${ }^{9}$ ) reported a significant difference was observed in changes in straight leg raising angles when the flexiondistraction technique was applied to patients with herniated intervertebral discs. Gionis and Groteke ${ }^{10}$ reported that $89 \%$ of 229 patients with symptoms associated with herniated intervertebral discs showed improvements when they underwent spinal decompression therapy for six weeks. According to Ma et al. ${ }^{11)}$, there was a statistically significant difference in changes in the straight leg raising test based on intervention periods when spinal decompression therapy with a therapeutic modality was applied to patients with herniated intervertebral discs for four weeks. In the present study, both groups also showed statistically significant differences. These results suggest that both manual and spinal decompression therapies can decrease the internal pressure of the intervertebral disc, can increase the intervertebral height without stimulating fibers around the annulus fibrosus that are susceptible to pain, and can realign the vertebral joints to their physiologically movable locations to alleviate pain, thereby restoring normal posture and activating body functions ${ }^{12)}$.

As for the intervertebral disc space changed by the flexion-distraction technique, Gudavalli et al. ${ }^{13)}$ reported that the L5-S1 disc space increased by approximately $3 \mathrm{~mm}$ and the angle of the intervertebral disc by around 6 degrees in the lumbar vertebrae of cadavers. When Gay et al. ${ }^{14)}$ measured the nucleus pulposus pressure and the annulus fibrosus compressive stress of 15 cadavers after the flexion-distraction technique, the technique decreased the pressure within the intervertebral disc by $65 \%$. According to $\mathrm{Cox}^{12}$, the intervertebral disc space enlarged by $1.5-2 \mathrm{~mm}$ during the flexion-distraction technique when investigated by X-ray. Similarly, both the spinal decompression therapy group and the manual therapy group in this study showed statistically significant differences. These results may indicate that the flexion-distraction technique enlarged the intervertebral disc space, formed a negative pressure within the disc, and extended the annulus fibrosus and posterior ligaments to alleviate nerve root adhesion and disc herniation ${ }^{9}$. Meanwhile, a chiropractic adjustment can realign the paravertebral tissues (muscles, ligaments, and facet joints) and the subluxation that stimulates the receptors within the spine, thereby activating the nerve reflex centers within the spinal cord or higher centers ${ }^{15}$.

There are some limitations to this study. For instance, the number of the patients was insufficient because they were patients who had visited our clinic for eight weeks; the lumbar lesions were restricted; we could not completely control the daily lives of the patients; and a long-term treatment was not provided. Further studies may be needed to either minimize or investigate the impact of these limitations from various perspectives.

\section{Conflict of interest}

None.

Table 1. Comparison of the straight leg raising angle and intervertebral disc height within each group

\begin{tabular}{llcc}
\hline & Group & Pre-treatment & Post-treatment \\
\hline SLRA (degrees) & EG & $53.5 \pm 10.7$ & $72.0 \pm 9.5^{* *}$ \\
& CG & $53.6 \pm 10.2$ & $71.7 \pm 7.9^{* *}$ \\
IDH $(\mathrm{mm})$ & EG & $14.7 \pm 2.3$ & $16.0 \pm 1.8^{* *}$ \\
& CG & $15.3 \pm 2.1$ & $16.3 \pm 2.3^{* *}$ \\
\hline
\end{tabular}

EG: experimental group; CG: control group.

$* * \mathrm{p}<0.01$, paired t-test. 


\section{REFERENCES}

1) Sims JA, Moorman SJ: The role of the iliolumbar ligament in low back pain. Med Hypotheses, 1996, 46: 511-515. [Medline] [CrossRef]

2) Hammer CJ: Chiropractic management and rehabilitation of a 38-year-old male with an L5-s1 disc herniation. J Chiropr Med, 2004, 3: 145-152. [Medline] [CrossRef]

3) Kim EG, Lee DG, Yoon EH, et al.: The clinical effects of sciatica with cox flexion distraction. The Journal of Korea CHUNA Manual Medicine for Spine \& Nerves, 2009, 4: 43-52.

4) Saravanakumar K, Harvey A: Lumbar zygapophyseal (facet) joint pain. Rev Pain, 2008, 2: 8-13. [Medline] [CrossRef]

5) Gay RE, Bronfort G, Evans RL: Distraction manipulation of the lumbar spine: a review of the literature. J Manipulative Physiol Ther, 2005, 28: 266-273. [Medline] [CrossRef]

6) Park CH: Clinical chiropractic manual. Seoul: Daekungbook publishing company, 2011, pp 273-274.

7) Hertzman-Miller RP, Morgenstern H, Hurwitz EL, et al.: Comparing the satisfaction of low back pain patients randomized to receive medical or chiropractic care: results from the UCLA low-back pain study. Am J Public Health, 2002, 92: 1628-1633. [Medline] [CrossRef]

8) Inoue H, Ohmori K, Miyasaka K, et al.: Radiographic evaluation of the lumbosacral disc height. Skeletal Radiol, 1999, 28: 638-643. [Medline] [CrossRef]

9) Kwon WA, Ryu YS, Ma SY.: The effects of Cox distraction manipulation on functional assessment measures and disc herniation index in patients with L4-5 herniated disc. Journal of the Korean Data \& Information Science Society, 2012, 23: 727-738. [CrossRef]

10) Gionis TA, Groteke E: Spinal decompression. Orthopedic technology review, 2003, 5, 36-39.

11) Ma SY, Kwon WA, Lee JH, et al.: The effects of spinal decompression combined with therapeutic modalities for patients with lumbar radiculopathy. Journal of the Korea Academia-Industrial Cooperation. Society, 2013, 14: 336-343.

12) Cox JM: Low back pain: mechanism, diagnosis and treatment. Lippincott Williams \& Wilkins, 2012, pp 167-168.

13) Gudavalli MR, Cambron JA, McGregor M, et al.: A randomized clinical trial and subgroup analysis to compare flexion-distraction with active exercise for chronic low back pain. Eur Spine J, 2006, 15: 1070-1082. [Medline] [CrossRef]

14) Gay RE, Ilharreborde B, Zhao KD, et al.: Stress in lumbar intervertebral discs during distraction: a cadaveric study. Spine J, 2008, 8: 982-990. [Medline] [CrossRef]

15) Haldeman S: Neurological effects of the adjustment. J Manipulative Physiol Ther, 2000, 23: 112-114. [Medline] [CrossRef] 